\title{
Clinicopathological factors in bladder cancer for cancer-specific survival outcomes following radical cystectomy: a systematic review and meta-analysis
}

\author{
Lijin Zhang ${ }^{1 *}$, Bin Wu' ${ }^{1}$ Zhenlei Zha', Wei Qu², Hu Zhao ${ }^{1}$ and Jun Yuan ${ }^{1}$
}

\begin{abstract}
Background: Assessing the prognostic significance of specific clinicopathological features plays an important role in surgical management after radical cystectomy. This study investigated the association between ten clinicopathological characteristics and cancer-specific survival (CSS) in patients with bladder cancer.

Methods: In accordance with the Preferred Reporting Items for Systematic Reviews and Meta-analyses (PRISMA) guidelines, a literature search was conducted through the PubMed, EMBASE and Web of Science databases using appropriate search terms from the dates of inception until November 2018. Pooled hazard ratios (HRs) with 95\% confidence intervals (Cls) were calculated to evaluate the CSS. Fixed- or random-effects models were constructed according to existence of heterogeneity.

Results: Thirty-three articles met the eligibility criteria for this systematic review, which included 19,702 patients. The overall results revealed that CSS was associated with advanced age (old vs. young: pooled $\mathrm{HR}=1.01 ; 95 \% \mathrm{Cl}$ : 1.00-1.01; $P<0.001$ ), higher tumor grade (3 vs. 1/2: pooled HR=1.29; 95\% Cl:1.15-1.45; $P<0.001$ ), higher pathological stage (3/4 vs. 1/2: pooled $H R=1.60 ; 95 \%$ Cl:1.37-1.86; $P<0.001$ ), lymph node metastasis (positive vs. negative: pooled $H R=1.51 ; 95 \% \mathrm{Cl}: 1.37-1.67 ; \mathrm{P}<0.001$ ), lymphovascular invasion (positive vs. negative: pooled $H R=$ 1.36; 95\% Cl:1.28-1.45; P < 0.001), and soft tissue surgical margin (positive vs. negative: pooled HR=1.42; 95\% Cl: 1.30-1.56; $P<0.001$ ). However, gender (male vs. female: pooled $H R=0.98 ; 95 \% \mathrm{Cl}: 0.96-1.01 ; P=0.278$ ), carcinoma in situ (positive vs. negative: pooled HR=0.98; 95\% Cl: 0.88-1.10; $P=0.753$ ), histology (transitional cell cancer vs variant: pooled $\mathrm{HR}=0.90 ; 95 \% \mathrm{Cl}: 0.79-1.02 ; P=0.089$ ) and adjuvant chemotherapy (yes vs. no: pooled $\mathrm{HR}=1.16$; 95\% Cl: $1.00-1.34 ; P=0.054)$ did not affect CSS after radical resection of bladder cancer.

Conclusions: Our results revealed that several clinicopathological characteristics can predict CSS risk after radical cystectomy. Prospective studies are needed to further confirm the predictive value of these variables for the prognosis of bladder cancer patients after radical cystectomy.
\end{abstract}

Keywords: Bladder cancer, Radical cystectomy, Cancer-specific survival, Meta-analysis

\footnotetext{
*Correspondence: stzlj913729553@163.com

'Department of Urology, Affiliated Jiang-yin Hospital of the Southeast

University Medical College, Jiang-yin 214400, China

Full list of author information is available at the end of the article
}

(C) The Author(s). 2019 Open Access This article is distributed under the terms of the Creative Commons Attribution 4.0 International License (http://creativecommons.org/licenses/by/4.0/), which permits unrestricted use, distribution, and reproduction in any medium, provided you give appropriate credit to the original author(s) and the source, provide a link to the Creative Commons license, and indicate if changes were made. The Creative Commons Public Domain Dedication waiver (http://creativecommons.org/publicdomain/zero/1.0/) applies to the data made available in this article, unless otherwise stated. 


\section{Background}

Bladder cancer $(\mathrm{BCa})$ is the most common malignancy of the urinary tract and occurs with a relatively high incidence in developing countries [1], with annual mortality rates ranging from approximately $1-5$ deaths per 100,000 men and $0.5-1.5$ deaths per 100,000 women [2]. Radical cystectomy $(\mathrm{RC})$ with bilateral pelvic lymph node dissection is the gold standard for patients with localized muscle-invasive tumors. Despite a better understanding of BCa biology and the use of adjuvant therapies, $\mathrm{BCa}$ continues to have high mortality rates, and the oncological outcomes following $\mathrm{RC}$ have not changed in the last 30 years [3].

$\mathrm{BCa}$ prognoses vary widely. Many factors have been investigated as potential predictors of clinical outcome in BCa. Positive soft tissue surgical margins (STSM) [4], lymphovascular invasion (LVI) [5], lymph node metastasis (LNM) [6], concomitant carcinoma in situ (CIS) [7], and failure to receive adjuvant chemotherapy (ACT) [8] have been reported to be associated with poor prognoses for $\mathrm{BCa}$ after RC. Although these predictive variables have contributed to estimating the $\mathrm{BCa}$ recurrence risk and survival outcomes, additional variables that can integrate with well-established prognostic models and provide accurate risk grading for $\mathrm{BCa}$ patients after $\mathrm{RC}$ are critical.

A major problem for urologists is identifying prognostic factors that can predict cancer progression. The ability to determine cancer-specific survival (CSS) and provide integrated patient survivorship and better estimates of survival probability at each follow-up may lead to more informative prognostic information in patient monitoring [9]. Therefore, we aimed to provide a comprehensive systematic review and metaanalysis of previous studies to investigate the prognostic roles of pathological status and clinical variables for CSS in patients following RC. We identified ten common clinicopathological characteristics that should be systematically assessed to guide postoperative decision-making after $\mathrm{RC}$.

\section{Methods}

\section{Search strategy}

In line with the guidelines of Preferred Reporting Items for Systematic Reviews and Meta-analyses (PRISMA) [10], the electronic database of PubMed, EMBASE and Web of Science were searched for studies published prior to November 2018. The following search term combinations were used: 'urinary bladder neoplasms', 'bladder and neoplasms', 'radical cystectomy', 'cancer-specific survival', 'clinical', and 'pathological'. The publication language was restricted to English. In addition, the reference lists of the identified studies were also searched manually.

\section{Inclusion and exclusion criteria}

The inclusion criteria were as follows: (1) all patients with BCa were pathologically confirmed; (2) the study included prognostic factors for CSS following radical cystectomy; (3) treatment was limited to $\mathrm{RC}$ in all studies; and (4) the authors provided the hazard ratios (HRs) and 95\% confidence intervals(CIs). The exclusion criteria were: (1) duplicates; (2) lack of sufficient data (HRs and CIs) for further analysis; and (3) case reports, reviews, letters, author replies, expert opinions or meeting abstracts. If the data overlapped across several different articles, only the most recent and informative article was selected.

\section{Data extraction and qualitative assessment}

Two authors extracted the information from the selected studies. Any disagreement between the reviewers was resolved by discussion with a third author. The following information were collected from eligible studies: first author's name, publication date, country, recruitment period, follow-up time, sample size, patient's age, pathological stage, tumor grade, histopathological subtype in transitional cell cancer (TCC) and the HR and 95\% CIs for CSS.

We evaluated the study quality using the 9-star Newcastle-Ottawa Scale (NOS) [11]. Scores of 7-9 indicated a high-quality study, and scores $<7$ indicated a low-quality. The cohort study quality was assessed as follows: object selection, inter-group comparability, and outcome measurement. Dichotomous variables were presented as HRs with 95\% CIs. If the data results were calculated by multivariate and univariate analysis simultaneously, the multivariate analyses were used.

\section{Statistical analysis}

All calculations were performed using STATA 12.0 software (Stata Corp LP, College Station, TX, USA). Heterogeneity was estimated using the Higgins Isquared statistic test, and $P_{\text {heterogeneity }} \leq 0.1$ or $I^{2}>$ $50 \%$. indicated heterogeneity among studies. When significant heterogeneity was observed among the studies, a random-effect (RE) model was used; otherwise, we adopted a fixed-effect (FE) model. To explore the source of heterogeneity, subgroup analysis was performed for CSS. Sensitivity analysis was conducted by excluding single studies one by one to examine the stability and reliability of the pooled results. A funnel plot and Egger's test were used to statistically evaluate the publication bias between studies. Two-tailed $P<0.05$ was considered statistically significant. 


\section{Results}

Literature search

From the search criteria, 887 articles were identified from the databases and the manual search. Of these articles, 664 studies were excluded based on their titles and/or abstracts, resulting in 223 studies for further analysis. The full texts were then screened, and 190 papers were excluded because of insufficient survival information or duplicated cohorts. Finally, 33 studies $[3,5,6,8,12-40]$ containing 19,702 patients (range 51-2,944) were included as per the eligibility criteria. Figure 1 presents a flowchart of the study selection process.

\section{Characteristics of eligible studies}

Tables 1 and 2 summarize the main characteristics and clinicopathological outcomes of the 33 included studies. All studies were performed retrospectively, and all were published between 2007 and 2018. Of the included studies, 11 were conducted in Asia, 8 in Europe, 7 in North America, 4 at international multicenters, 3 in
Turkey and 1 in Australia. Histopathological examinations were performed on resected tumor specimens. All studies used CSS as a common endpoint to evaluate the prognostic value of the clinicopathological indicators of survival. The quality scores of the studies ranged from 7 to 9.Therefore, all included studies were of high quality (studies with a score $\geq 7$; Additional file 2: Table S1).

\section{Meta-analysis}

Our meta-analysis demonstrated that advanced age (old vs. young: pooled $\mathrm{HR}=1.01 ; 95 \% \mathrm{CI}$ : $1.00-1.01 ; P<$ $0.001 ; I^{2}=68.2 \%, P_{\text {heterogeneity }}<0.001 ;$ Fig. 2A), higher tumor grade ( 3 vs. $1 / 2$ : pooled $\mathrm{HR}=1.29$; $95 \% \mathrm{CI}$ : $1.15-$ $1.45 ; P_{\text {heterogeneity }}<0.001 ; I^{2}=76.9 \%, P_{\text {heterogeneity }}<0.001$; Fig. $2 \mathrm{~B})$, higher pathological stage $(3 / 4$ vs. $1 / 2$ : pooled $\mathrm{HR}=1.60 ; 95 \%$ CI: $1.37-1.86 ; P<0.001 ; I^{2}=92.2 \%, P_{h e-}$ terogeneity $<0.001$; Fig. 2C), LNM (positive vs. negative: pooled HR $=1.51 ; 95 \%$ CI: $1.37-1.67 ; P_{\text {heterogeneity }}<0.001$; $I^{2}=95 \%, P<0.001$; Fig. 2D), LVI (positive vs. negative: pooled $\mathrm{HR}=1.36 ; 95 \% \mathrm{CI}: 1.28-1.45 ; \mathrm{P}<0.001 ; I^{2}=$

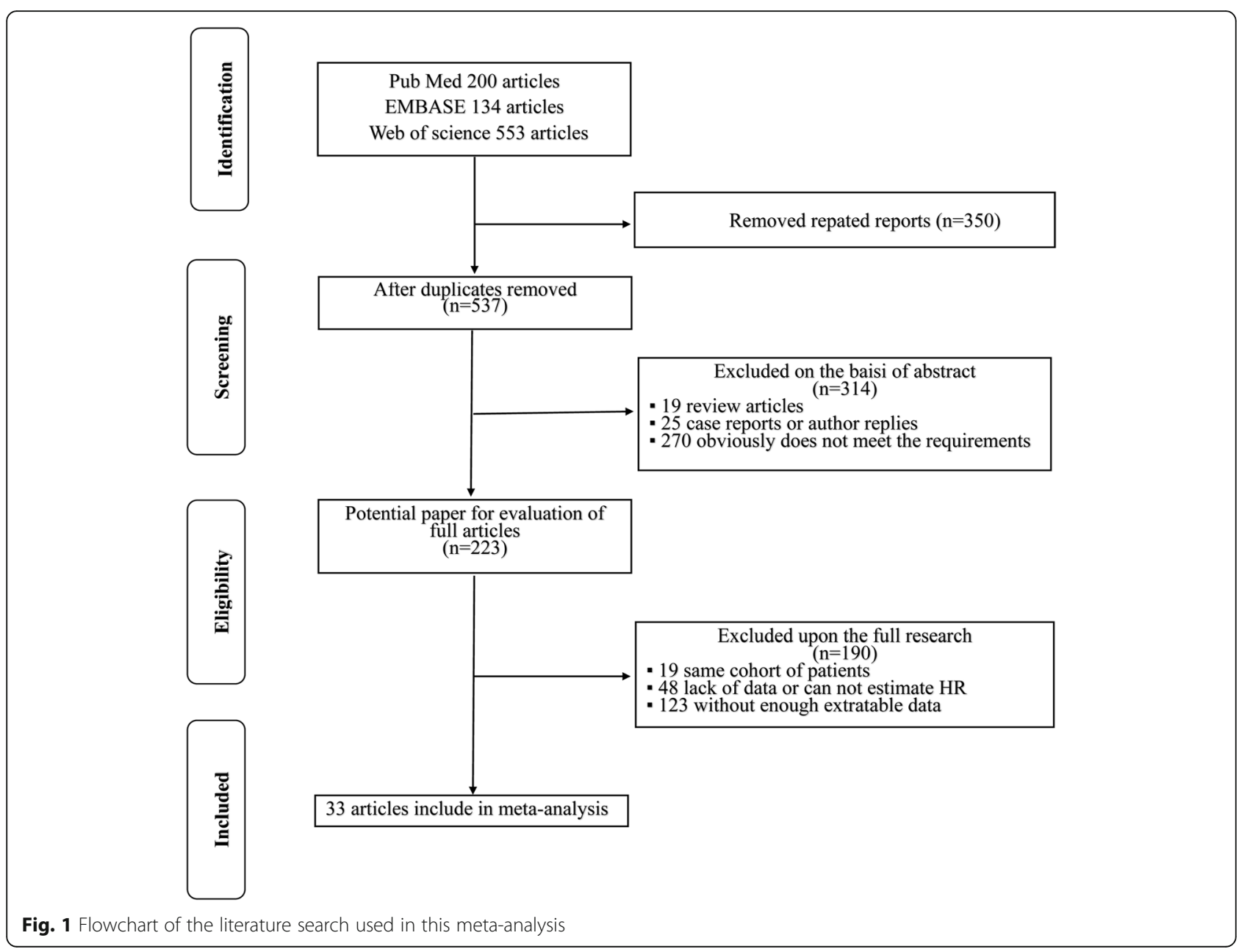




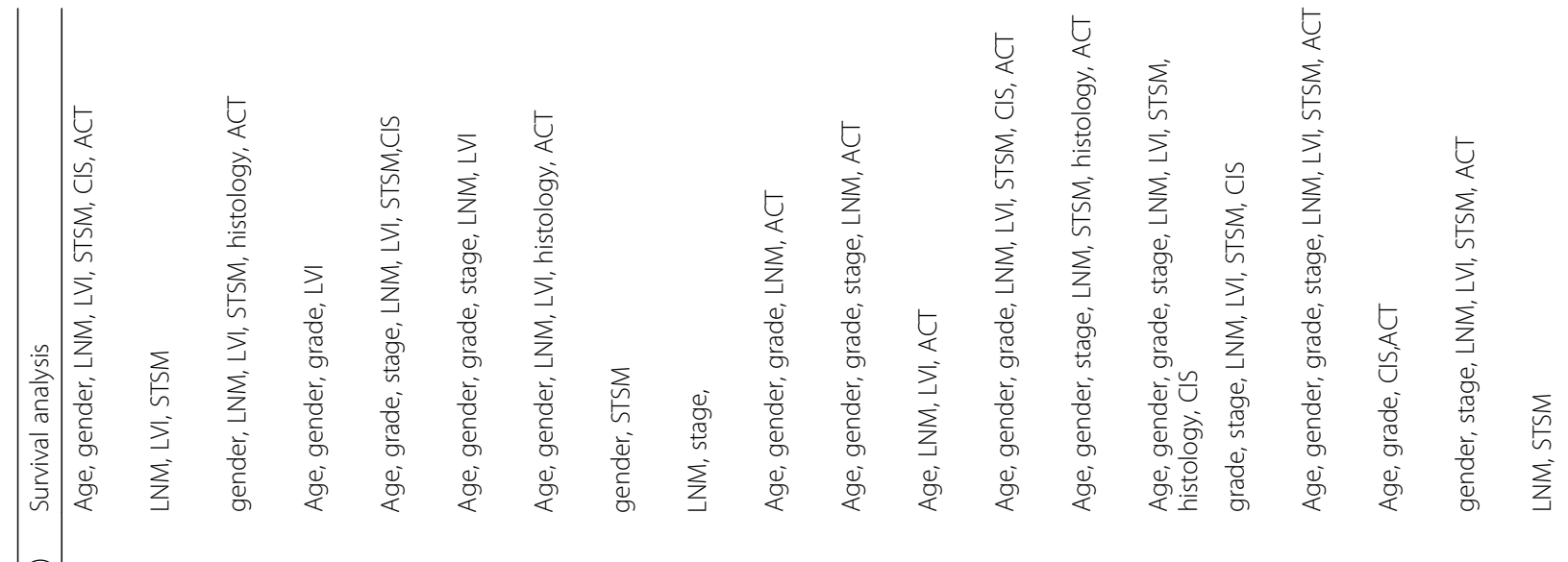

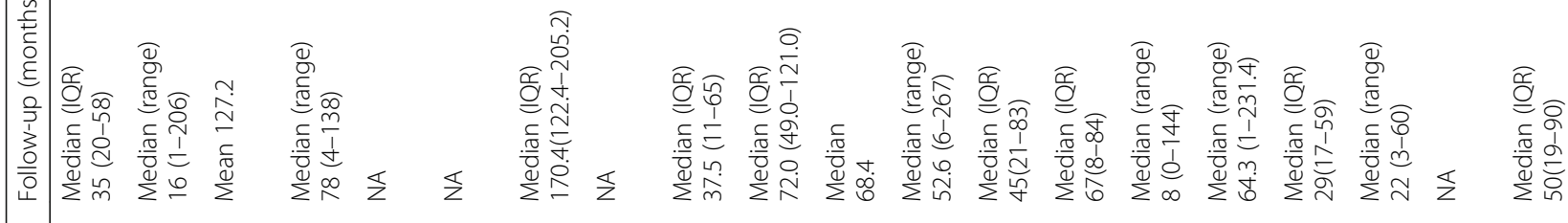

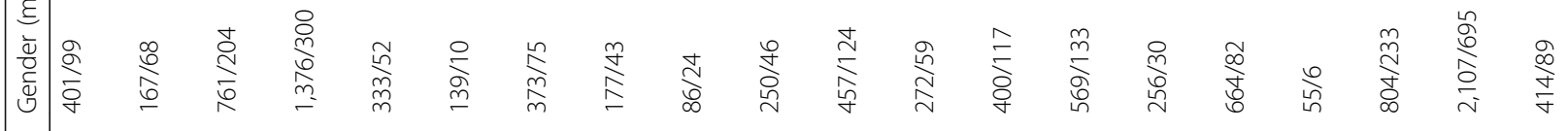

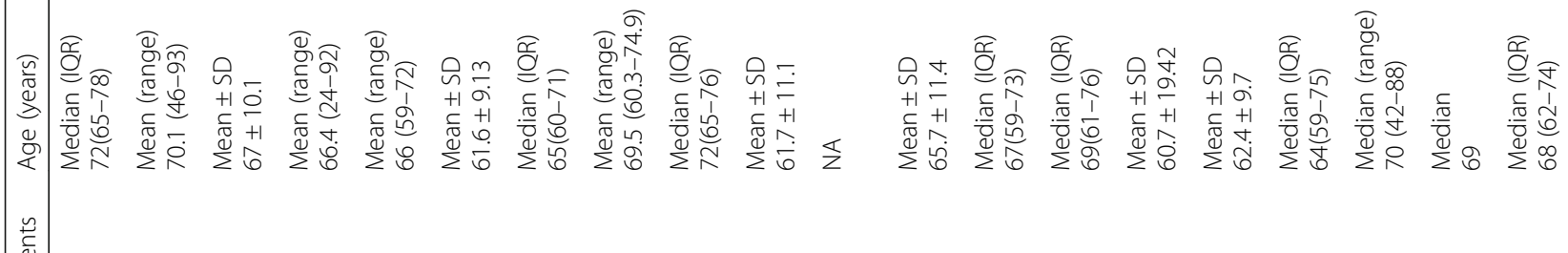

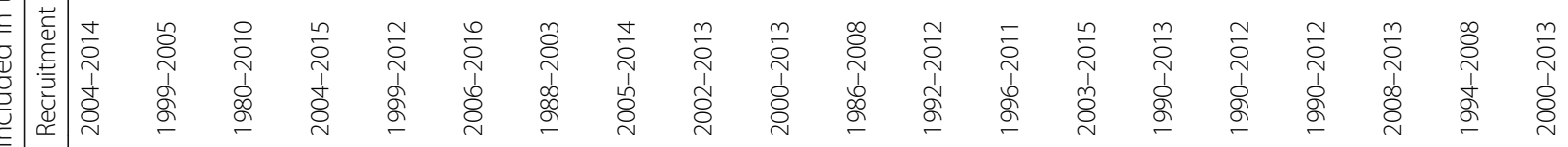

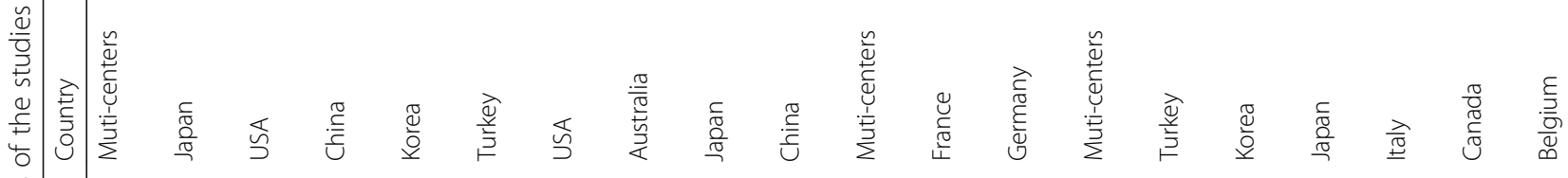

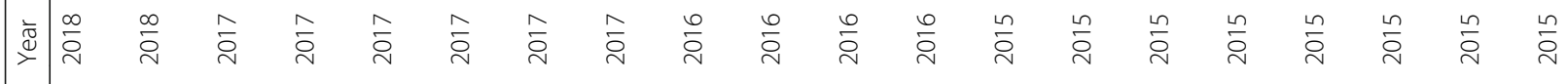

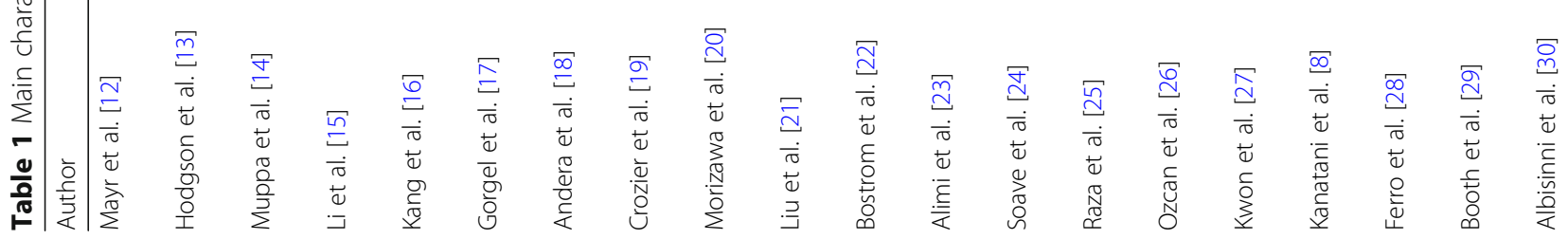




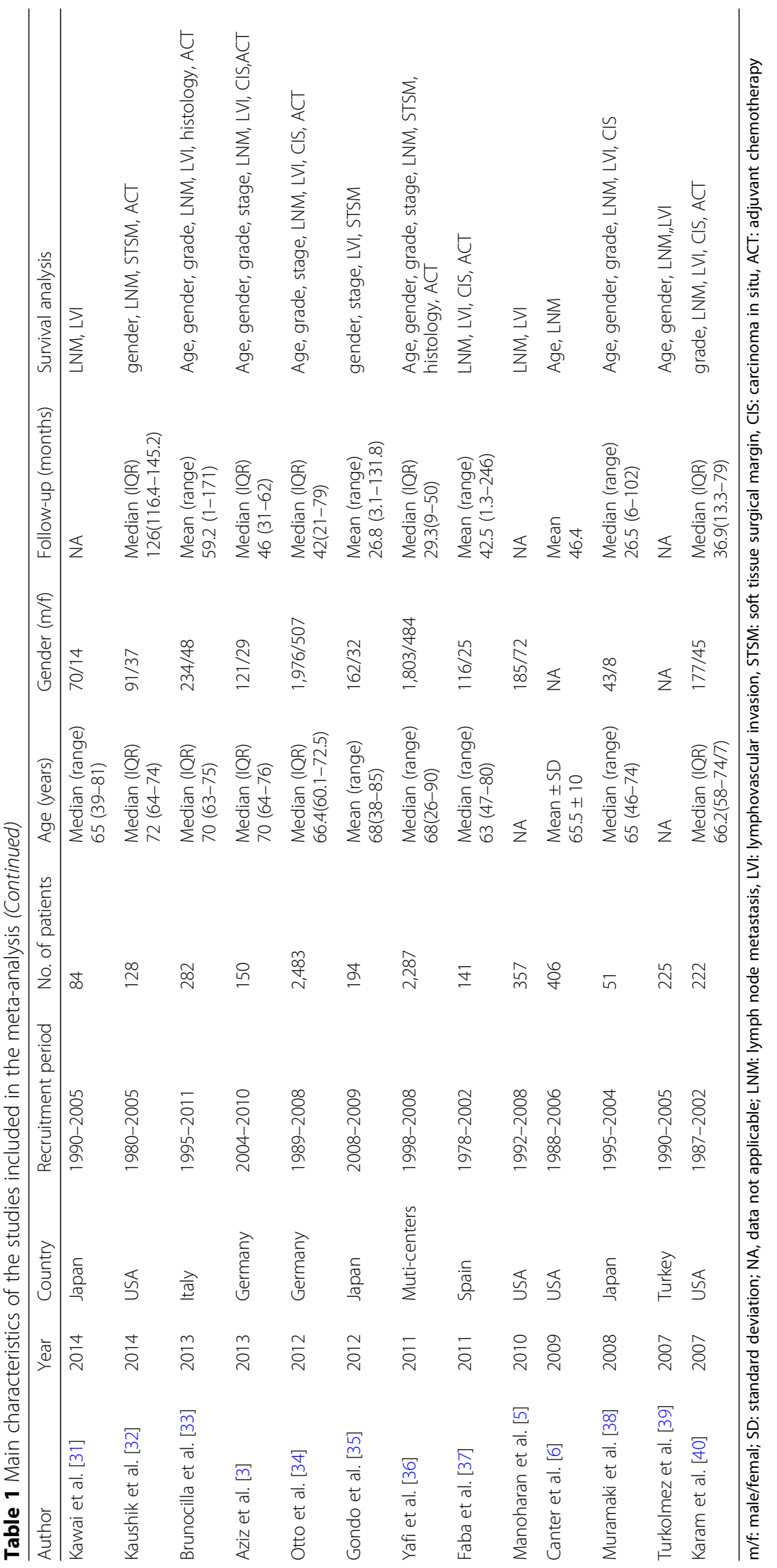


Table 2 Tumor characteristics of all studies included in the meta-analysis

\begin{tabular}{|c|c|c|c|c|c|c|c|c|c|}
\hline Study & $\begin{array}{l}\text { Staging } \\
\text { system }\end{array}$ & $\begin{array}{l}\text { Grading } \\
\text { system }\end{array}$ & $\begin{array}{l}\text { LNM + } \\
\text { / LNM - }\end{array}$ & $\begin{array}{l}\mathrm{ClS}+ \\
/ \mathrm{ClS}-\end{array}$ & $\begin{array}{l}\text { Stage 1-2/ } \\
3-4\end{array}$ & $\begin{array}{l}\text { Grade } \\
1-2 / 3\end{array}$ & $\begin{array}{l}\text { STSM +/ } \\
\text { STSM- }\end{array}$ & LVI+/ LVI- & $\begin{array}{l}\text { ACT administered/ } \\
\text { no ACT }\end{array}$ \\
\hline Mayr et al. [12] & 2010 TNM & NA & $132 / 368$ & $171 / 329$ & $276 / 224$ & NA & $47 / 453$ & $200 / 300$ & $65 / 435$ \\
\hline Hodgson et al. [13] & 2010 AJCC & WHO & $89 / 146$ & $107 / 128$ & $46 / 189$ & NA & $58 / 177$ & $149 / 86$ & $47 / 188$ \\
\hline Muppa et al. [14] & 2010 AJCC & $\mathrm{WHO}$ & $797 / 168$ & NA & $536 / 429$ & NA & $23 / 942$ & $306 / 659$ & NA \\
\hline Li et al. [15] & 2009 TNM & WHO & NA & NA & $1,676 / 0$ & $685 / 991$ & NA & $188 / 1,488$ & NA \\
\hline Kang et al. [16] & 2009 TNM & WHO/ISUP & $191 / 46$ & $78 / 159$ & $168 / 69$ & $51 / 185$ & $3 / 234$ & $67 / 170$ & $185 / 52$ \\
\hline Gorgel et al. [17] & 2009 TNM & WHO & $53 / 96$ & NA & $74 / 75$ & $29 / 119$ & NA & $44 / 105$ & NA \\
\hline Andera et al. [18] & 2009 TNM & WHO & $277 / 171$ & NA & $160 / 288$ & $12 / 436$ & NA & $185 / 163$ & $40 / 408$ \\
\hline Crozier et al. [19] & 2009 TNM & NA & NA & NA & $155 / 65$ & NA & $17 / 203$ & NA & NA \\
\hline Morizawa et al. [20] & 2009 TNM & WHO & $22 / 88$ & NA & $56 / 54$ & NA & 13/97 & $31 / 79$ & NA \\
\hline Liu et al. [21] & 2002 TNM & WHO & $63 / 233$ & NA & 194/102 & $75 / 221$ & NA & NA & $75 / 221$ \\
\hline Bostrom et al. [22] & 2002 TNM & WHO & $301 / 280$ & NA & $407 / 174$ & $109 / 472$ & NA & NA & $77 / 504$ \\
\hline Alimi et al. [23] & NA & NA & 195/136 & NA & 140/191 & NA & $40 / 291$ & NA & $11 / 320$ \\
\hline Soave et al. [24] & 2002 TNM & WHO & $138 / 379$ & $187 / 330$ & 0/293 & $30 / 263$ & $261 / 32$ & NA & $101 / 416$ \\
\hline Raza et al. [25] & 2002 TNM & $\mathrm{WHO}$ & $33 / 484$ & NA & $260 / 257$ & NA & $55 / 462$ & NA & $134 / 383$ \\
\hline Ozcan et al. [26] & 2002 TNM & WHO & $42 / 244$ & $19 / 267$ & $162 / 124$ & $96 / 190$ & $18 / 268$ & $51 / 235$ & NA \\
\hline Kwon et al. [27] & 2010 AJCC & $\mathrm{WHO}$ & $556 / 190$ & $189 / 557$ & $386 / 338$ & $108 / 636$ & $23 / 723$ & $310 / 436$ & $176 / 570$ \\
\hline Kanatani et al. [8] & 2009 AJCC & WHO & $18 / 43$ & NA & $8 / 53$ & $7 / 54$ & $7 / 54$ & $51 / 10$ & 61 \\
\hline Ferro et al. [28] & 2009 TNM & $\mathrm{WHO}$ & $266 / 771$ & $162 / 875$ & $813 / 224$ & $115 / 922$ & NA & NA & $301 / 736$ \\
\hline Booth et al. [29] & NA & NA & $821 / 2,123$ & NA & $807 / 1,995$ & NA & $377 / 2,567$ & $1,451 / 1,493$ & $537 / 2,407$ \\
\hline Albisinni et al. [30] & NA & NA & $387 / 116$ & NA & $291 / 212$ & NA & $29 / 474$ & NA & NA \\
\hline Kawai et al. [31] & NA & NA & $65 / 19$ & NA & NA & $21 / 60$ & NA & $49 / 35$ & NA \\
\hline Kaushik et al. [32] & 2010 TNM & WHO & $53 / 75$ & NA & $0 / 128$ & NA & $20 / 108$ & NA & NA \\
\hline Brunocilla et al. [33] & 2009 TNM & WHO & $207 / 75$ & NA & $147 / 135$ & $66 / 216$ & NA & $115 / 167$ & $91 / 191$ \\
\hline Aziz et al. [3] & 2009 TNM & WHO & $59 / 91$ & $72 / 78$ & $57 / 93$ & $11 / 139$ & NA & $85 / 65$ & $35 / 115$ \\
\hline Otto et al. [34] & 2002 TNM & ISUP & $640 / 1,843$ & $765 / 1,718$ & $1,377 / 1,106$ & $829 / 1,654$ & NA & $876 / 1,607$ & $245 / 2,138$ \\
\hline Gondo et al. [35] & NA & NA & $21 / 173$ & NA & $108 / 86$ & $21 / 173$ & $20 / 174$ & 99/95 & $48 / 146$ \\
\hline Yafi et al. [36] & 1997 TNM & WHO & $544 / 1,559$ & NA & $1,164 / 1,123$ & NA & $173 / 1,843$ & NA & $401 / 1,662$ \\
\hline Faba et al. [37] & 2002 AJCC & WHO & $7 / 134$ & $33 / 108$ & $141 / 0$ & $132 / 9$ & NA & $28 / 113$ & $15 / 126$ \\
\hline Manoharan et al. [5] & 1997 TNM & $\mathrm{WHO}$ & $73 / 284$ & $136 / 221$ & $224 / 133$ & $54 / 293$ & NA & $105 / 252$ & NA \\
\hline Canter et al. [6] & 1997 TNM & WHO & NA & NA & $368 / 38$ & NA & NA & $40 / 366$ & NA \\
\hline Muramaki et al. [38] & 2002 TNM & WHO & $26 / 25$ & $7 / 44$ & $6 / 45$ & $7 / 44$ & NA & $41 / 10$ & $51 / 0$ \\
\hline Turkolmez et al. [39] & 1997 TNM & WHO & $131 / 94$ & NA & $157 / 68$ & NA & NA & NA & NA \\
\hline Karam et al. [40] & 2002 TNM & WHO & $65 / 160$ & $93 / 132$ & $107 / 119$ & $17 / 209$ & NA & $101 / 124$ & $60 / 165$ \\
\hline
\end{tabular}

SD: standard deviation; NA: data not applicable; AJCC: American Joint Committee on Cancer classification; WHO/ ISUP: World Health Organization/International Society of Urological Pathology classification; LNM: Iymph node metastasis, LVI: lymphovascular invasion, STSM: soft tissue surgical margin, CIS: carcinoma in situ, ACT: adjuvant chemotherapy

68.4\%, $P_{\text {heterogeneity }}<0.001$; Fig. 2E), and STSM (positive vs. negative: pooled $\mathrm{HR}=1.42 ; 95 \% \mathrm{CI}: 1.30-1.56 ; \mathrm{P}<$ $0.001 ; I^{2}=71.7 \%, P_{\text {heterogeneity }}<0.001$; Fig. $2 \mathrm{~F}$ ) in $\mathrm{BCa}$ were associated with poor CSS. However, no significant correlations were observed regarding gender (male vs. female: pooled $\mathrm{HR}=0.98 ; 95 \% \mathrm{CI}: 0.96-1.01 ; P=0.278$; $I^{2}=34.9 \%, P_{\text {heterogeneity }}=0.036$; Fig. 3A), CIS (positive vs. negative: pooled $\mathrm{HR}=0.98$; $95 \% \mathrm{CI}$ : $0.88-1.10 ; P=0.753$;
$I^{2}=78 \%, P_{\text {heterogeneity }}<0.001$; Fig. 3B), histology (TCC vs variant: pooled $\mathrm{HR}=0.90 ; 95 \% \mathrm{CI}: 0.79-1.02 ; P=0.089$; $I^{2}=71.6 \%, P_{\text {heterogeneity }}=0.003$; Fig. $3 \mathrm{C}$ ) or ACT (yes vs. no: pooled HR $=1.16 ; 95 \%$ CI: $1.00-1.34 ; P=0.054 ; I^{2}=$ 93.8\%, $P_{\text {heterogeneity }}<0.001$; Fig. 3D).

To explore the source of heterogeneity for advanced age, tumor grade, pathological stage, LNM, LVI, STSM, CIS and ACT, their significance levels 


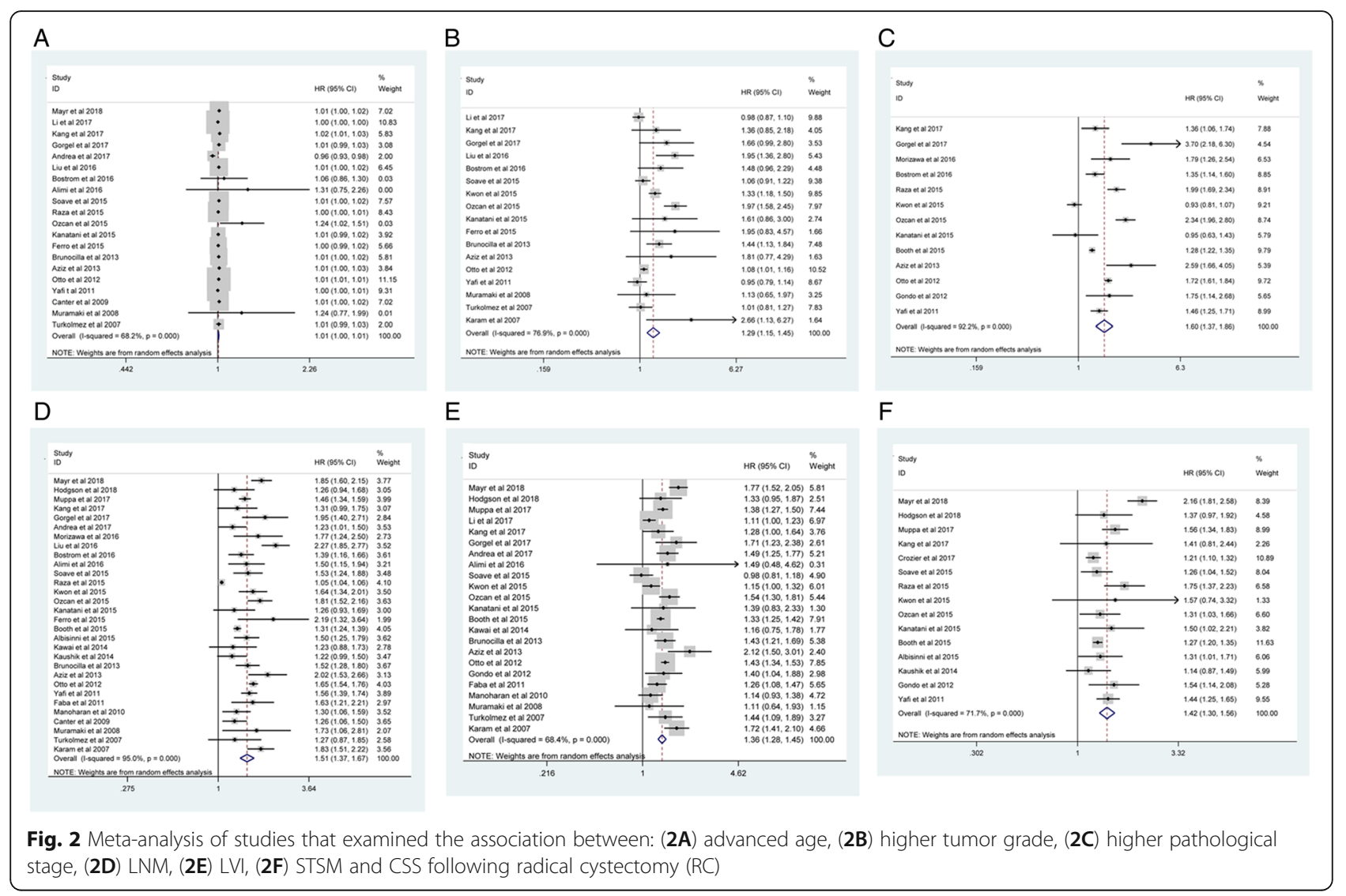

were further evaluated via subgroup analysis based on geographical region (Asia vs. non-Asia), year of publication ( $\geq 2015$ vs. $<2015)$, number of patients $(\geq 500$ vs. $<500)$ and median follow-up ( $\geq 36$ months vs. $<36$ months). Because few studies were included in the histology group, no subgroup analysis was conducted for histology. Table 3 presents the subgroup analysis results for CSS. Notably, we observed a significant decline in heterogeneity for CSS in some categories, such as in articles published before 2015, studies with sample sizes of $<500$ cases and median follow-ups of $<36$ months. The subgroup analysis results were consistent with the primary findings.

\section{Sensitivity analysis}

The pooled HR for CSS for advanced age ranged from 1.01 (95\% CI:1.00-1.01) to 1.01 (95\% CI:1.001.01), for gender ranged from 0.98 (95\% CI: 0.941.02 ) to 0.99 (95\% CI: $0.99-1.04)$, for tumor grade ranged from 1.25 (95\% CI: $1.11-1.41)$ to 1.34 (95\% CI: 1.16-1.54), for pathological stage ranged from 1.53 (95\% CI: $1.31-1.79$ ) to 1.68 (95\% CI: $1.45-1.95$ ), for LNM ranged from 1.49 (95\% CI: $1.35-1.64)$ to
1.52 (95\% CI: 1.37-1.68), for LVI ranged from 1.34 (95\% CI: $1.26-1.42$ ) to 1.38 (95\% CI: $1.30-1.47$ ), for STSM ranged from 1.34 (95\% CI: $1.26-1.43$ ) to 1.44 (95\% CI: 1.29-1.61), for CIS ranged from 0.95 (95\% CI: $0.86-1.05)$ to 1.01 (95\% CI: $0.89-1.14$ ), for histology ranged from 0.86 (95\% CI: $0.76-0.97)$ to 0.94 (95\% CI: 0.82-1.07), and for ACT ranged from 1.12 (95\% CI: $0.97-1.29)$ to 1.19 (95\% CI: $1.02-1.38$ ) (Additional file 1: Figure S1).These results indicated that our findings were reliable and robust.

\section{Publication bias}

Figure 4 shows the funnel plots for publication bias. Egger's test demonstrated that no publication bias existed regarding advanced age ( $\mathrm{p}$ Egger $=0.427$, Fig. 4A), gender (p Egger $=0.487$, Fig. 4B), CIS ( Egger $=0.172$, Fig. $4 C)$, LVI $(p$ Egger $=0.797$, Fig. 4D), pathological stage $(p$ Egger $=0.330$, Fig. 4E), STSM $(p$ Egger $=0.134$, Fig. $4 \mathrm{~F})$, histology $(\mathrm{p}$ Egger $=0.648$, Fig. 4G) and ATC (p Egger $=0.266$, Fig. 4H). However, publication biases were found for tumor grade ( $p$ Egger $=0.023$, Fig. 4I) and LNM (p Egger $<0.001$, Fig. $4 \mathrm{~J}$ ), suggesting that publication bias may have played a potential role in tumor grade and LNM. 


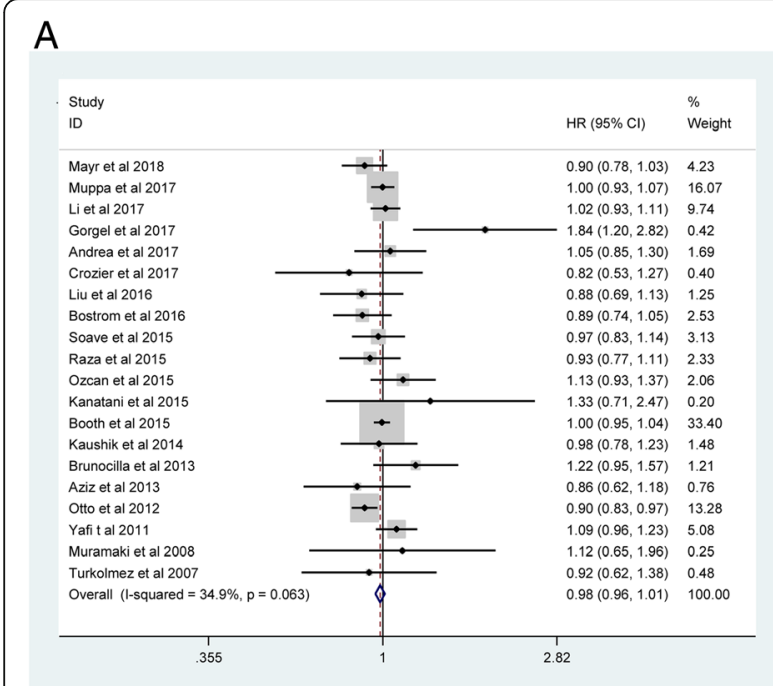

B

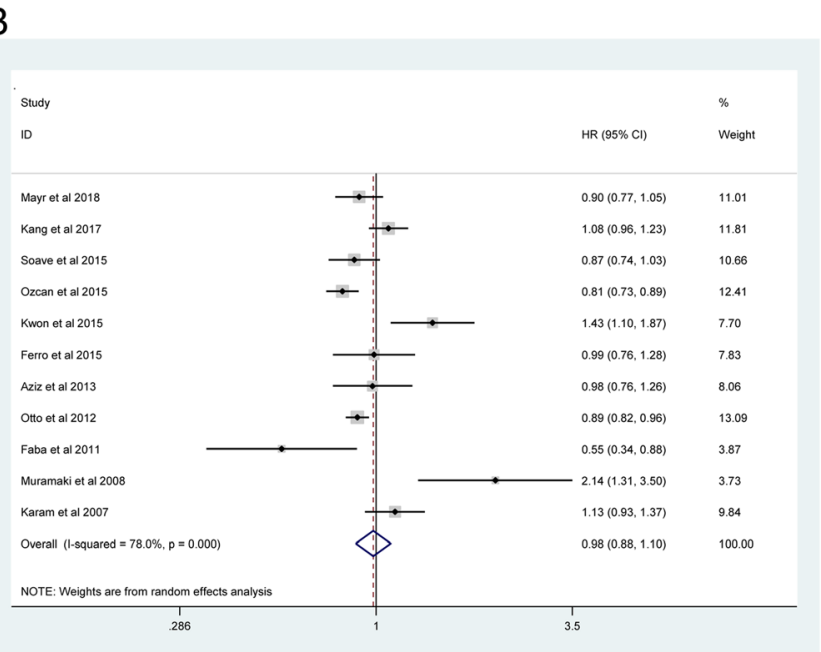

C

$\mathrm{D}$
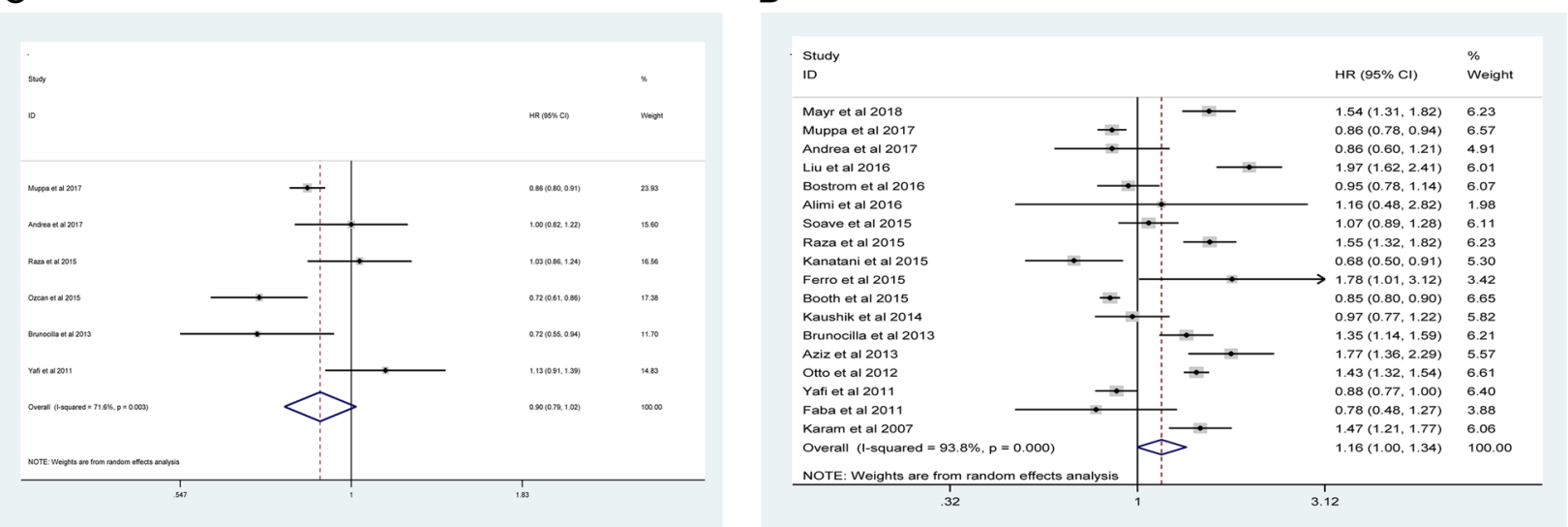

Fig. 3 Meta-analysis of studies that examined the association between: (3A) gender, (3B) CIS, (3C) histology, (3D) ACT and CSS following radical cystectomy (RC)

\section{Discussion}

Despite modern advancements in surgical techniques, the oncological outcomes of $\mathrm{BCa}$ remains poor. The 5 -yr overall survival rates were only $60 \%$ according to a multicenter database [41]. Determining the probability of CSS after RC is difficult because it can vary according to the different clinical features and various tumor characteristics. The traditional clinicopathological features, such as sex [34], pathological tumor stage or grade [25] and LNM [6], have been identified as important parameters with prognostic predictive value and contribute to postoperative clinical decision making based on some nomograms.

Currently, the TNM staging system, which is based on pathological tumor stage and grade, tumor histological subtype, and lymph node status [42] is the most commonly used preoperative model for predicting CSS in $\mathrm{BCa}$ patients. Another predictive model is the
European Organisation for the Research and Treatment of Cancer (EORTC) risk stratification scheme [43], which uses grade (World Health Organization [WHO] 1973), stage, CIS, multiplicity, size and previous recurrence rate to determine the risk of CSS after $\mathrm{RC}$. Although these two traditional prognostic models have been externally validated, significant variations were founded in some studies. Variations in tumor outcomes may have been related to the heterogeneity of $\mathrm{BCa}$ biology and different clinicopathological features in patients with BCa.

Tumor markers that can accurately predict the oncological outcomes in $\mathrm{BCa}$ patients when applied with other pathological parameters are essential for clinical decision making. Some published studies on molecular biomarkers, such as luminal and basal subtypes [44], the gene alterations nuclear matrix protein number 22 [45], and the bladder tumor antigen (BTA) stat test [46], have been 
Table 3 Summary and subgroup results of the association between common clinicopathological characteristics and BCa

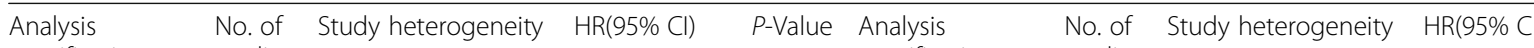

specification studies $\frac{\text { specification }}{P(\%) P_{\text {heterogeneity }}} \quad$ studies $\frac{P(\%) P_{\text {heterogeneity }}}{P^{2}}$

Advanced age LV

$\begin{array}{lllll}\text { Overall } & 20 & 68.2 & <0.001 & 1.01(1.00,1.01)\end{array}<0.001$

Overall 23

Geographical region

Geographical region

$\begin{array}{llllll}\text { Asia } & 8 & 59.3 & 0.016 & 1.01(1.00,1.02) & 0.023 \\ \text { non-Asia } & 12 & 68.5 & <0.001 & 1.01(1.00,1.01) & 0.004\end{array}$

Year of publication

$\begin{array}{llllll}\geq 2015 & 13 & 72.4 & <0.001 & 1.01(1.00,1.01) & 0.037 \\ <2015 & 7 & 39.4 & 0.129 & 1.01(1.00,1.01) & <0.001\end{array}$

No. of patients

$\begin{array}{llllll}\geq 500 & 8 & 71.9 & 0.001 & 1.01(1.00,1.01) & 0.002 \\ <500 & 12 & 65 & 0.001 & 1.01(1.00,1.02) & 0.074\end{array}$

Median follow-up

$$
\geq 36 \text { months } 8
$$

$<36$ months 9

Grade

Overall

Geographical region

$\begin{array}{ll}\text { Asia } & 9 \\ \text { non-Asia } & 8\end{array}$

Year of publication

$\begin{array}{llll}\geq 2015 & 10 & 81.6 & <0.001 \\ <2015 & 7 & 54.4 & 0.041\end{array}$

No. of patients

$\begin{array}{llll}\geq 500 & 7 & 71.1 & 0.002 \\ <500 & 10 & 60.5 & 0.007\end{array}$

Median follow-up

$$
\geq 36 \text { months } 6
$$

$<36$ months 8

Stage

Overall 13

Geographical region

$$
\begin{array}{ll}
\text { Asia } & 7 \\
& 5
\end{array}
$$

non-Asia 5

$$
\geq 2015 \quad 9
$$$$
<2015
$$

No. of patients

$\begin{array}{ll}\geq 500 & 8 \\ <500 & 5\end{array}$

$\begin{array}{ll}93.1 & <0.001 \\ 87.2 & <0.001\end{array}$

Median follow-up

$\geq 36$ months 4

$<36$ months 6

LNM

$\begin{array}{ll}74.8 & <0.001 \\ 35.5 & 0.134\end{array}$

$76.9<0.001$

$82.6<0.001$

$57.9 \quad 0.002$

$1.17(1.03,1.34) \quad 0.020$

$1.00(0.99,1.01) \quad 0.736$

$1.01(1.00,1.01)<0.001$

Asia 11

non-Asia

12

Year of publication

No. of patients

Median follow-up

$\geq 36$ months 7

$<36$ months 10

STSM

$1.29(1.15,1.45)<0.001 \quad$ Overall 15

Geographical region

Year of publication

No. of patients

$\begin{array}{ll}1.41(1.17,1.70) & <0.001 \\ 1.13(0.98,1.31) & 0.085\end{array}$

$\geq 500$

$1.11(0.99,1.23) \quad 0.072$

$<500$

10
5

$\geq 36$ months 6

CIS

Overall

Geographical region

$\begin{array}{llll}93.1<0.001 \quad 1.61(1.10,2.63) & 0.013\end{array}$

Asi

non-Asia

Year of publication

$$
92.7<0.001 \quad 1.54(1.25,1.90)<0.001
$$

$\geq 2015$

$<2015$

No. of patients

$$
\begin{array}{ll}
1.47(1.24,1.73) & <0.001 \\
1.92(1.29,2.87) & 0.001
\end{array}
$$$$
\geq 500
$$$$
<500
$$

Median follow-up

$\begin{array}{ll}96.4 & <0.001 \\ 65.9 & 0.012\end{array}$

$\begin{array}{ll}1.55(1.02,2.37) & 0.042 \\ 1.62(1.37,1.92) & <0.001\end{array}$

$\geq 36$ months 2

$<36$ months 8

ACT

$\begin{array}{llllll}\geq 2015 & 13 & 74.8 & <0.001 & 1.34(1.22,1.46) & <0.001 \\ <2015 & 10 & 48.9 & 0.040 & 1.40(1.28,1.54) & <0.001\end{array}$

$\begin{array}{llllll}\geq 500 & 10 & 80.6 & <0.001 & 1.30(1.19,1.42) & <0.001 \\ <500 & 13 & 39.1 & 0.073 & 1.44(1.32,1.57) & <0.001\end{array}$

$\begin{array}{llllll}\text { Asia } & 7 & 0 & 0.650 & 1.26(1.17,1.36) & <0.001 \\ \text { non-Asia } & 8 & 55.5 & <0.001 & 1.46(1.27,1.67) & <0.001\end{array}$

$\begin{array}{llllll}\geq 2015 & 12 & 76.1 & <0.001 & 1.44(1.29,1.61) & <0.001 \\ <2015 & 3 & 29.3 & 0.243 & 1.38(1.19,1.60) & <0.001\end{array}$

Median follow-up

$<36$ months 6 
Table 3 Summary and subgroup results of the association between common clinicopathological characteristics and BCa (Continued)

\begin{tabular}{|c|c|c|c|c|c|c|c|c|c|c|c|}
\hline \multirow{3}{*}{$\begin{array}{l}\text { Analysis } \\
\text { specification }\end{array}$} & \multirow{3}{*}{$\begin{array}{l}\begin{array}{l}\text { No. of } \\
\text { studies }\end{array} \\
30\end{array}$} & \multirow{2}{*}{\multicolumn{2}{|c|}{$\frac{\text { Study heterogeneity }}{P(\%) P_{\text {heterogeneity }}}$}} & \multirow{3}{*}{$\begin{array}{l}\mathrm{HR}(95 \% \mathrm{Cl}) \\
1.51(1.37,1.67)\end{array}$} & \multirow{3}{*}{$\begin{array}{l}P \text {-Value } \\
<0.001\end{array}$} & \multirow{3}{*}{$\begin{array}{l}\begin{array}{l}\text { Analysis } \\
\text { specification }\end{array} \\
\text { Overall }\end{array}$} & \multirow{3}{*}{$\begin{array}{l}\begin{array}{l}\text { No. of } \\
\text { studies }\end{array} \\
18\end{array}$} & \multirow{2}{*}{\multicolumn{2}{|c|}{$\frac{\text { Study heterogeneity }}{P^{2}(\%) P_{\text {heterogeneity }}}$}} & \multirow{3}{*}{$\begin{array}{l}\mathrm{HR}(95 \% \mathrm{Cl}) \\
1.16(1.00,1.34)\end{array}$} & \multirow{3}{*}{$\frac{P \text {-Value }}{0.054}$} \\
\hline & & & & & & & & & & & \\
\hline & & 95 & $<0.001$ & & & & & 93.8 & $<0.001$ & & \\
\hline \multicolumn{6}{|c|}{ Geographical region } & \multicolumn{6}{|c|}{ Geographical region } \\
\hline Asia & 11 & 61.2 & 0.004 & $1.58(1.38,1.81)$ & $<0.001$ & Asia & 2 & 97.1 & $<0.001$ & $1.16(0.41,3.31)$ & 0.775 \\
\hline non-Asia & 19 & 96.2 & $<0.001$ & $1.48(1.32,1.66)$ & $<0.001$ & non-Asia & 16 & 93.4 & $<0.001$ & $1.15(0.99,1.34)$ & 0.063 \\
\hline \multicolumn{6}{|c|}{ Year of publication } & \multicolumn{6}{|c|}{ Year of publication } \\
\hline$\geq 2015$ & 18 & 94.9 & $<0.001$ & $1.52(1.34,1.71)$ & $<0.001$ & $\geq 2015$ & 11 & 93.4 & $<0.001$ & $1.12(0.92,1.37)$ & 0.243 \\
\hline$<2015$ & 12 & 58.6 & 0.005 & $1.50(1.38,1.64)$ & $<0.001$ & $<2015$ & 7 & 89.6 & $<0.001$ & $1.21(0.99,1.48)$ & 0.053 \\
\hline \multicolumn{6}{|l|}{ No. of patients } & \multicolumn{6}{|l|}{ No. of patients } \\
\hline$\geq 500$ & 14 & 98.9 & $<0.001$ & $1.48(1.29,1.70)$ & $<0.001$ & $\geq 500$ & 9 & 95.7 & $<0.001$ & $1.13(0.94,1.37)$ & 0.201 \\
\hline$<500$ & 16 & 69.1 & $<0.001$ & $1.53(1.38,1.71)$ & $<0.001$ & $<500$ & 9 & 86.3 & $<0.001$ & $1.18(0.93,1.50)$ & 0.177 \\
\hline \multicolumn{6}{|l|}{ Median follow-up } & \multicolumn{6}{|l|}{ Median follow-up } \\
\hline$\geq 36$ months & 11 & 95.3 & $<0.001$ & $1.47(1.24,1.74)$ & $<0.001$ & $\geq 36$ months & 8 & 92.4 & $<0.001$ & $1.16(0.91,1.49)$ & 0.228 \\
\hline$<36$ months & 13 & 49.4 & 0.022 & $1.61(1.49,1.74)$ & $<0.001$ & $<36$ months & 9 & 89.9 & $<0.001$ & $1.20(0.99,1.46)$ & 0.065 \\
\hline
\end{tabular}

adopted in recent years to improve diagnosing and managing patients receiving RC. However, none of these biomarkers have been shown to be sufficiently sensitive or specific in predicting survival outcomes. Therefore, in this study, we exploited more validated prognostic factors, including clinical variables (age, gender), pathological information (tumor stage and grade, LNM and STSM, LVI, CIS, and histology), and whether adjuvant therapy (ACT) was received for predicting CSS in BCa patients.

This is the first study to systematically assess the association between ten clinicopathological features and CSS of BCa in a single study. To improve the statistical power and provide more credible results, 33 cohort studies with a large combined sample size of $19,702 \mathrm{BCa}$ patients who underwent RC were pooled in our study. Strictly adhering to the inclusion and exclusion criteria, we extracted the raw data from the relevant studies. The results revealed that advanced age, higher tumor grade, LNM, LVI, and positive STSM significantly predicted the CSS of BCa patients (all $P \leq 0.05$ ). Hence, these clinicopathological findings were independent risk factors in this meta-analysis. Besieds, all the results were reliable and robust via the subgroup and sensitivity analyses.

Interestingly, our results indicated that gender, CIS, histology and ACT may not be associated with CSS. Studies on gender, histology and CIS as prognostic factors for $\mathrm{BCa}$ patients have stimulated considerable interest, but the results remain controversial and ambiguous for managing $\mathrm{BCa}$. Some investigators reported that gender and CIS had independent prognostic significance $[14,34,47]$, while others considered that gender and CIS may not be significant factors in determining terminal prognosis compared with other widely used prognostic indicators [18, 48, 49]. Additionally, administering ACT after RC in patients with high-risk $\mathrm{BCa}$ remains a challenge for clinical urologists. Despite numerous studies being published, no level 1 evidence has demonstrated that ATC confers a significant survival benefit to $\mathrm{BCa}$ patients after $\mathrm{RC}$ [50]. In the present study, rigorous data analysis indicated that these three factors may not affect the CSS prognosis of patients with $\mathrm{BCa}$.

Although this was a comprehensive meta-analysis, the present study had several limitations. First, most included studies were retrospective cohort studies, and data extracted from those studies may have led to inherent bias. Thus, a prospective multicenter trial providing more definite answers is needed. Second, substantial heterogeneity was observed in some studies. Although we found no possible source of heterogeneity after several subgroup analyses, the conclusions drawn from this meta-analysis should be approached with caution. However, the pooled results in most of the subgroup analyses were consistent with the overall findings. Third, the studies retrieved for our analysis were limited to those published in English, which may result in a language bias. Studies with negative results are not often published in English-language journals [51]; thus, our research may contain some publication bias.

\section{Conclusions}

In summary, the data from this meta-analysis indicate that $\mathrm{BCa}$ patients with advanced age, higher tumor grade, LNM, LVI, and positive STSM are likely to have poorer CSS, suggesting that these parameters may be independent indicators of $\mathrm{BCa}$ in patients following $\mathrm{RC}$. In 
A

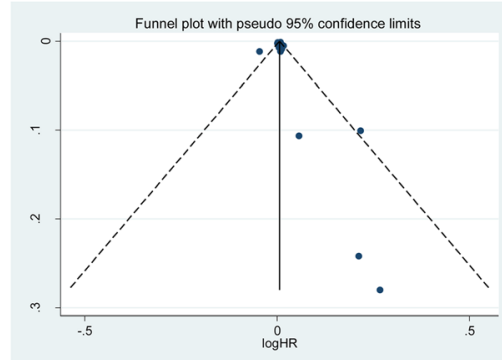

C

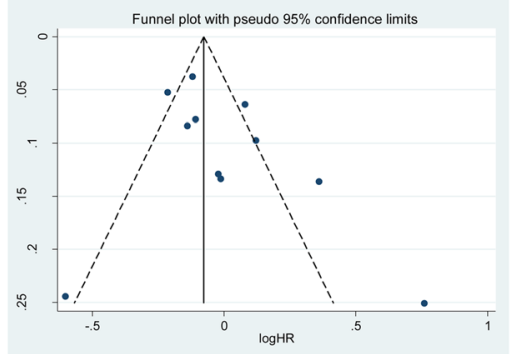

E

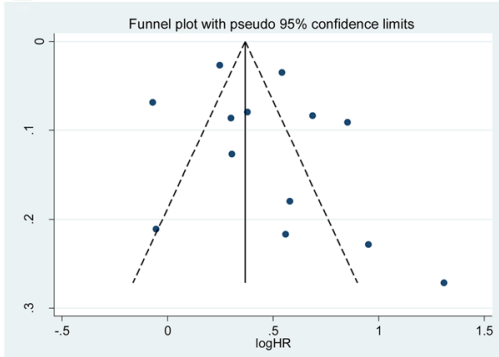

G

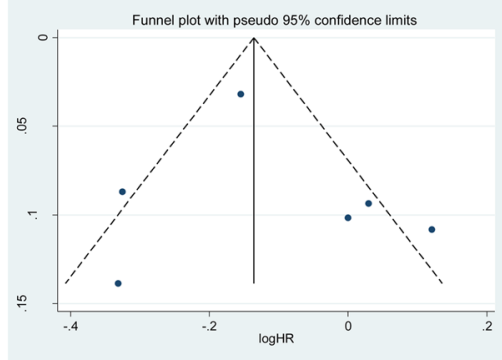

I

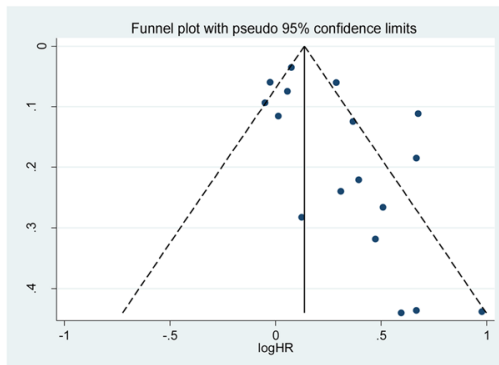

B

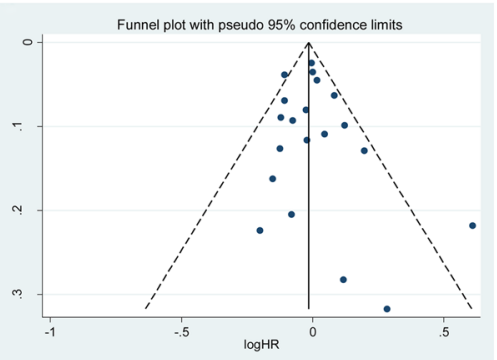

D

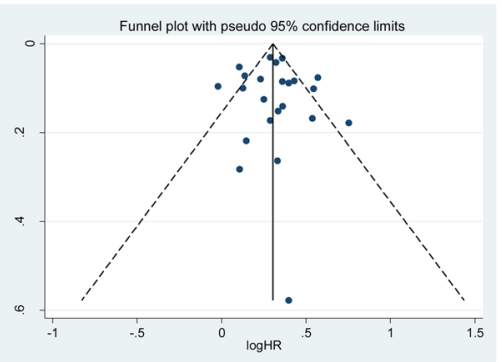

F

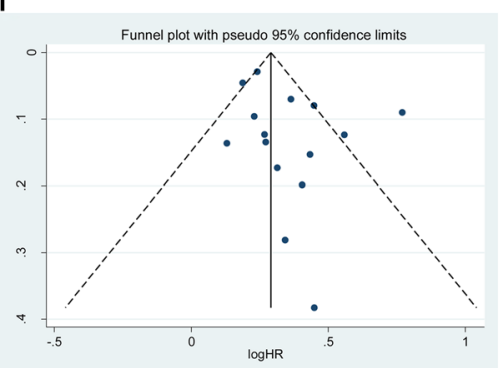

$\mathrm{H}$

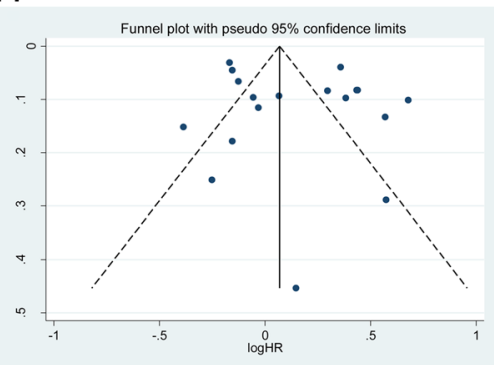

J

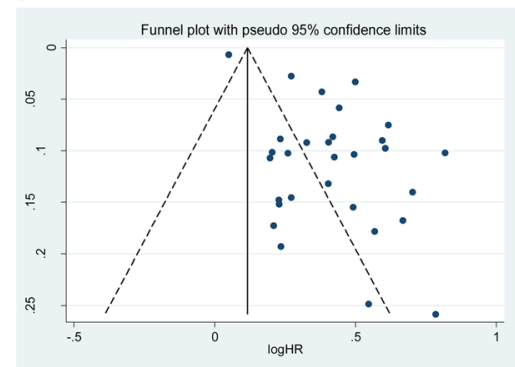

Fig. 4 Funnel plots for the publication bias test. Each point represents a separate study for the indicated association. The vertical line represents the mean effects size: (4A) advanced age; (4B) gender; (4C) CIS; (4D) LVI; (4E) pathological stage; (4F) STSM; (4G) histology; (4H) ATC; (4I) tumor grade and (4 J) LNM 
contrast with what is seen clinical practice, gender, CIS, histology and postoperative ACT were not predictors of CSS in patients with BCa. We identified significant patient-specific (age) and tumor-specific (higher tumor grade, LNM, LVI, and positive STSM) predictors of CSS to propose a risk-based strategy for choosing surveillance and postoperative treatment options. Despite our rigorous systematic approach, further large, prospective studies are needed to confirm our findings considering the inherent limitations of the included studies.

\section{Additional files}

Additional file 1: Figure S1 Sensitivity analysis for: (S1A) advanced age; (S1B) gender; (S1C) tumor grade; (S1D) pathological stage; (S1E) LNM; (S1F) LVI; (S1G) STSM; (S1H) CIS; (S1I) histology; (S1J) ACT. (TIF 10703 kb)

Additional file 2: Table S1 Quality assessment of the cohort studies included in this meta-analysis. (DOCX $57 \mathrm{~kb}$ )

\section{Abbreviations}

ACT: Adjuvant chemotherapy; BCa: Bladder cancer; CIS: Concomitant carcinoma in situ; Cls: Confidence intervals; CSS: Cancer-specific survival; EORTC: European Organisation for the Research and Treatment of Cancer; HRs: Hazard ratios; LNM: Lymph node metastasis; LVI: Lymphovascular invasion; NOS: Newcastle-Ottawa scale; PRISMA: Preferred Reporting Items for Systematic Reviews and Meta-Analyses; RC: Radical cystectomy; STSM: Soft tissue surgical margin; TCC: Transitional cell cancer

\section{Authors' contributions}

LZ: Project development and Manuscript writing; BW: Data Collection and Manuscript editing; ZLZ: Data Collection; WQ: Data Management; HZ: Data analysis, Data Management; JY: Data analysis, Data Management. All authors approved the final manuscript.

\section{Funding}

No funding was received for this study.

\section{Availability of data and materials}

All data generated or analyzed during the present study are included in this published article (and its supplementary information files).

\section{Ethics approval and consent to participate}

Not applicable.

\section{Consent for publication}

Not applicable.

\section{Competing interests}

We declare that there are no potential competing interests in this research.

\section{Author details}

'Department of Urology, Affiliated Jiang-yin Hospital of the Southeast University Medical College, Jiang-yin 214400, China. ${ }^{2}$ Department of Pharmacy, Affiliated Jiang-yin Hospital of the Southeast University Medical College, Jiang-yin 214400, China.

Received: 16 January 2019 Accepted: 11 July 2019

Published online: 19 July 2019

\section{References}

1. Jemal A, Bray F, Center MM, Ferlay J, Ward E, Forman D. Global cancer statistics. CA Cancer J Clin. 2011;61(2):69-90.

2. Burger M, Catto JW, Dalbagni G, Grossman HB, Herr H, Karakiewicz P, Kassouf W, Kiemeney LA, La Vecchia C, Shariat S, et al. Epidemiology and risk factors of urothelial bladder cancer. Eur Urol. 2013;63(2):234-41.
3. Aziz A, Gierth M, Fritsche HM, May M, Otto W, Denzinger S, Wieland WF, Merseburger A, Riedmiller $H$, Kocot A, et al. Oncological outcome of primary versus secondary muscle-invasive bladder cancer is comparable after radical cystectomy. Urol Int. 2013;91(1):97-102.

4. Sharma P, Zargar-Shoshtari K, Poch MA, Pow-Sang JM, Sexton WJ, Spiess PE, Gilbert SM. Surgical control and margin status after robotic and open cystectomy in high-risk cases: caution or equivalence? World J Urol. 2017; 35(4):657-63.

5. Manoharan M, Katkoori D, Kishore TA, Jorda M, Luongo T, Soloway MS. Lymphovascular invasion in radical cystectomy specimen: is it an independent prognostic factor in patients without lymph node metastases? World J Urol. 2010;28(2):233-7.

6. Canter D, Guzzo TJ, Resnick MJ, Bergey MR, Sonnad SS, Tomaszewski J, VanArsdalen K, Malkowicz SB. A thorough pelvic lymph node dissection in presence of positive margins associated with better clinical outcomes in radical cystectomy patients. Urology. 2009;74(1):161-5.

7. Yafi FA, Aprikian AG, Chin JL, Fradet Y, Izawa J, Estey E, Fairey A, Rendon R, Cagiannos I, Lacombe L, et al. Impact of concomitant carcinoma in situ on upstaging and outcome following radical cystectomy for bladder cancer. World J Urol. 2014;32(5):1295-301.

8. Kanatani A, Nakagawa T, Kawai T, Naito A, Sato Y, Yoshida K, Nozaki K, Nagata M, Yamada Y, Azuma T, et al. Adjuvant chemotherapy is possibly beneficial for locally advanced or node-positive bladder cancer. Clinical genitourinary cancer. 2015;13(2):e107-12.

9. Sun M, Abdollah F, Bianchi M, Trinh QD, Shariat SF, Jeldres C, Tian Z, Hansen J, Briganti A, Graefen M et al: Conditional survival of patients with urothelial carcinoma of the urinary bladder treated with radical cystectomy. European journal of cancer (Oxford, England: 1990) 2012, 48(10):1503-1511.

10. Liberati A, Altman DG, Tetzlaff J, Mulrow C, Gotzsche PC, loannidis JP, Clarke M, Devereaux PJ, Kleijnen J, Moher D. The PRISMA statement for reporting systematic reviews and meta-analyses of studies that evaluate health care interventions: explanation and elaboration. J Clin Epidemiol. 2009;62(10):e1-34.

11. Stang A. Critical evaluation of the Newcastle-Ottawa scale for the assessment of the quality of nonrandomized studies in meta-analyses. Eur J Epidemiol. 2010;25(9):603-5.

12. Mayr R, Gierth M, Zeman F, Reiffen M, Seeger P, Wezel F, Pycha A, Comploj E, Bonatti M, Ritter M, et al. Sarcopenia as a comorbidity-independent predictor of survival following radical cystectomy for bladder cancer. J Cachexia Sarcopenia Muscle. 2018;9(3):505-13.

13. Hodgson A, Xu B, Satkunasivam R, Downes MR. Tumour front inflammation and necrosis are independent prognostic predictors in high-grade urothelial carcinoma of the bladder. J Clin Pathol. 2018;71(2):154-60.

14. Muppa P, Gupta S, Frank I, Boorjian SA, Karnes RJ, Thompson RH, Thapa P, Tarrell RF, Herrera Hernandez LP, Jimenez RE, et al. Prognostic significance of lymphatic, vascular and perineural invasion for bladder cancer patients treated by radical cystectomy. Pathology. 2017;49(3):259-66.

15. Li G, Yu J, Song H, Zhu S, Sun L, Shang Z, Niu Y. Squamous differentiation in patients with superficial bladder urothelial carcinoma is associated with high risk of recurrence and poor survival. BMC Cancer. 2017;17(1):530.

16. Kang M, Balpukov UJ, Jeong CW, Kwak C, Kim HH, Ku JH. Can the preoperative neutrophil-to-lymphocyte ratio significantly predict the conditional survival probability in muscle-invasive bladder Cancer patients undergoing radical cystectomy? Clinical genitourinary cancer. 2017;15(3): e411-20.

17. Gorgel SN, Kose O, Koc EM, Ates E, Akin Y, Yilmaz Y. The prognostic significance of preoperatively assessed AST/ALT (De Ritis) ratio on survival in patients underwent radical cystectomy. Int Urol Nephrol. 2017;49(9):1577-83.

18. D'Andrea D, Moschini M, Soria F, Gust KM, Briganti A, Karakiewicz PI, Roupret M, Shariat SF. ABO blood group and rhesus factor are not associated with outcomes after radical cystectomy for non-metastatic urothelial carcinoma of the bladder. Anticancer Res. 2017;37(10):5747-53.

19. Crozier J, Papa N, Perera M, Stewart M, Goad J, Sengupta S, Bolton D, Lawrentschuk N. Lymph node yield in node-negative patients predicts cancer specific survival following radical cystectomy for transitional cell carcinoma. Investigative and clinical urology. 2017;58(6):416-22.

20. Morizawa Y, Miyake M, Shimada K, Hori S, Tatsumi Y, Nakai Y, Anai S, Tanaka N, Konishi N, Fujimoto K. Neutrophil-to-lymphocyte ratio as a detection marker of tumor recurrence in patients with muscle-invasive bladder cancer after radical cystectomy. Urologic oncology. 2016;34(6):257.e211-57.

21. Liu J, Dai Y, Zhou F, Long Z, Li Y, Liu B, Xie D, Tang J, Tan J, Yao K, et al. The prognostic role of preoperative serum albumin/globulin ratio in patients 
with bladder urothelial carcinoma undergoing radical cystectomy. Urologic oncology. 2016;34(11):484.e481-8.

22. Bostrom PJ, Mirtti T, van Rhijn B, Fleshner NE, Finelli A, Laato M, Jewett MA, Moore MJ, Sridhar S, Nurmi M, et al. Benefit of adjuvant chemotherapy and pelvic lymph node dissection in PT3 and node positive bladder Cancer patients treated with radical cystectomy. Bladder cancer. 2016;2(2):263-72.

23. Alimi Q, Verhoest G, Kammerer-Jacquet SF, Mathieu R, Rioux-Leclercq N, Manunta A, Laguerre B, Guille F, Bensalah K, Peyronnet B. Role of routine computed tomography scan in the oncological follow up of patients treated by radical cystectomy for bladder cancer. International journal of urology : official journal of the Japanese Urological Association. 2016;23(10):840-6.

24. Soave A, John LM, Dahlem R, Minner S, Engel O, Schmidt S, Kluth LA, Fisch M, Rink M. The impact of tumor diameter and tumor necrosis on oncologic outcomes in patients with urothelial carcinoma of the bladder treated with radical cystectomy. Urology. 2015;86(1):92-8.

25. Raza SJ, Wilson T, Peabody JO, Wiklund P, Scherr DS, Al-Daghmin A, Dibaj S, Khan MS, Dasgupta P, Mottrie A, et al. Long-term oncologic outcomes following robot-assisted radical cystectomy: results from the international robotic cystectomy consortium. Eur Urol. 2015;68(4):721-8.

26. Ozcan C, Telli O, Ozturk E, Suer E, Gokce MI, Gulpinar O, Oztuna D, Baltaci S, Gogus C: The prognostic significance of preoperative leukocytosis and neutrophil-to-lymphocyte ratio in patients who underwent radical cystectomy for bladder cancer. Canadian Urological Association journal = Journal de I'Association des urologues du Canada 2015, 9(11-12):E789-E794.

27. Kwon T, Jeong IG, Lee J, Lee C, You D, Hong B, Hong JH, Ahn H, Kim CS. Adjuvant chemotherapy after radical cystectomy for bladder cancer: a comparative study using inverse-probability-of-treatment weighting. J Cancer Res Clin Oncol. 2015;141(1):169-76.

28. Ferro M, De Cobelli O, Buonerba C, Di Lorenzo G, Capece M, Bruzzese D, Autorino R, Bottero D, Cioffi A, Matei DV, et al. Modified Glasgow prognostic score is associated with risk of recurrence in bladder Cancer patients after radical cystectomy: a multicenter experience. Medicine. 2015;94(42):e1861.

29. Booth CM, Siemens DR, Wei X, Peng Y, Berman DM, Mackillop WJ. Pathological factors associated with survival benefit from adjuvant chemotherapy (ACT): a population-based study of bladder cancer. BJU Int. 2015;116(3):373-81.

30. Albisinni S, Rassweiler J, Abbou CC, Cathelineau X, Chlosta P, Fossion L, Gaboardi F, Rimington P, Salomon L, Sanchez-Salas R, et al. Long-term analysis of oncological outcomes after laparoscopic radical cystectomy in Europe: results from a multicentre study by the European Association of Urology (EAU) section of Uro-technology. BJU Int. 2015;115(6):937-45.

31. Kawai T, Enomoto Y, Morikawa T, Matsushita H, Kume H, Fukayama M, Yamaguchi H, Kakimi K, Homma Y. High expression of heat shock protein 105 predicts a favorable prognosis for patients with urinary bladder cancer treated with radical cystectomy. Molecular and clinical oncology. 2014;2(1):38-42.

32. Kaushik D, Frank I, Eisenberg MS, Cheville JC, Tarrell R, Thapa P, Thompson RH, Boorjian SA. Gender-specific survival following radical cystectomy for pT4 bladder cancer. World J Urol. 2014;32(6):1433-9.

33. Brunocilla E, Pernetti R, Schiavina R, Borghesi M, Vagnoni V, Rocca GC, Borgatti F, Concetti S, Martorana $\mathrm{G}$. The number of nodes removed as well as the template of the dissection is independently correlated to cancerspecific survival after radical cystectomy for muscle-invasive bladder cancer. Int Urol Nephrol. 2013:45(3):711-9.

34. Otto W, May M, Fritsche HM, Dragun D, Aziz A, Gierth M, Trojan L, Herrmann E, Moritz R, Ellinger J, et al. Analysis of sex differences in cancerspecific survival and perioperative mortality following radical cystectomy: results of a large German multicenter study of nearly 2500 patients with urothelial carcinoma of the bladder. Gend Med. 2012;9(6):481-9.

35. Gondo T, Nakashima J, Ozu C, Ohno Y, Horiguchi Y, Namiki K, Yoshioka K, Ohori M, Hatano T, Tachibana M. Risk stratification of survival by lymphovascular invasion, pathological stage, and surgical margin in patients with bladder cancer treated with radical cystectomy. Int J Clin Oncol. 2012;17(5):456-61.

36. Yafi FA, Aprikian AG, Chin JL, Fradet Y, Izawa J, Estey E, Fairey A, Rendon R, Cagiannos I, Lacombe L, et al. Contemporary outcomes of 2287 patients with bladder cancer who were treated with radical cystectomy: a Canadian multicentre experience. BJU Int. 2011;108(4):539-45.

37. Rodriguez Faba O, Palou J, Rosales A, Breda A, Algaba F, Urdaneta G, Villavicencio $\mathrm{H}$. Clinical predictive factors of poor outcome in patients with stage pT0 disease at radical cystectomy. J Urol. 2011;186(2):442-7.

38. Muramaki M, Miyake H, Kurahashi T, Takenaka A, Inoue TA, Fujisawa M. Prognostic significance of adjuvant cisplatin-based combination chemotherapy following radical cystectomy in patients with invasive bladder cancer. International journal of urology : official journal of the Japanese Urological Association. 2008;15(4):314-8.

39. Turkolmez K, Tokgoz H, Resorlu B, Kose K, Beduk Y. Muscle-invasive bladder cancer: predictive factors and prognostic difference between primary and progressive tumors. Urology. 2007;70(3):477-81.

40. Karam JA, Lotan Y, Karakiewicz Pl, Ashfaq R, Sagalowsky Al, Roehrborn CG, Shariat SF. Use of combined apoptosis biomarkers for prediction of bladder cancer recurrence and mortality after radical cystectomy. The Lancet Oncology. 2007:8(2):128-36.

41. Stein JP, Lieskovsky G, Cote R, Groshen S, Feng AC, Boyd S, Skinner E, Bochner B, Thangathurai D, Mikhail M, et al. Radical cystectomy in the treatment of invasive bladder cancer: long-term results in 1,054 patients. Journal of clinical oncology : official journal of the American Society of Clinical Oncology. 2001;19(3):666-75.

42. Tilki D, Reich O, Karakiewicz PI, Novara G, Kassouf W, Ergun S, Fradet Y, Ficarra V, Sonpavde G, Stief CG, et al. Validation of the AJCC TNM substaging of pT2 bladder cancer: deep muscle invasion is associated with significantly worse outcome. Eur Urol. 2010;58(1):112-7.

43. Rieken M, Shariat SF, Kluth L, Crivelli JJ, Abufaraj M, Foerster B, Mari A, llijazi D, Karakiewicz PI, Babjuk M, et al. Comparison of the EORTC tables and the EAU categories for risk stratification of patients with nonmuscle-invasive bladder cancer. Urologic oncology. 2018;36(1):8.e17-18.e24.

44. Warrick Jl, Kaag M, Raman JD, Chan W, Tran T, Kunchala S, Shuman L, DeGraff D, Chen G. FOXA1 and CK14 as markers of luminal and basal subtypes in histologic variants of bladder cancer and their associated conventional urothelial carcinoma. Virchows Archiv : an international journal of pathology. 2017:471(3):337-45

45. Shariat SF, Savage C, Chromecki TF, Sun M, Scherr DS, Lee RK, Lughezzani G, Remzi M, Marberger MJ, Karakiewicz Pl, et al. Assessing the clinical benefit of nuclear matrix protein 22 in the surveillance of patients with nonmuscleinvasive bladder cancer and negative cytology: a decision-curve analysis. Cancer. 2011;117(13):2892-7.

46. Guo A, Wang X, Gao L, Shi J, Sun C, Wan Z. Bladder tumour antigen (BTA stat) test compared to the urine cytology in the diagnosis of bladder cancer: A meta-analysis. Canadian Urological Association journal = Journal de I'Association des urologues du Canada. 2014;8(5-6):E347-52.

47. Moschini M, Shariat SF, Abufaraj M, Soria F, Klatte T, Croce G, Mattei A, Damiano R, Salonia A, Montorsi F, et al. The presence of carcinoma in situ at radical cystectomy increases the risk of urothelial recurrence: Implications for follow-up schemes. Urologic oncology. 2017;35(4):151.e117-23.

48. Nuhn P, Bastian PJ, Novara G, Svatek RS, Karakiewicz PI, Skinner E, Fradet Y, Izawa Jl, Kassouf W, Montorsi F, et al. Concomitant carcinoma in situ in cystectomy specimens is not associated with clinical outcomes after surgery. Urol Int. 2011;87(1):42-8.

49. Scosyrev E, Golijanin D, Wu G, Messing E. The burden of bladder cancer in men and women: analysis of the years of life lost. BJU Int. 2012;109(1):57-62.

50. Raghavan D, Bawtinhimer A, Mahoney J, Eckrich S, Riggs S. Adjuvant chemotherapy for bladder cancer-why does level 1 evidence not support it? Annals of oncology : official journal of the European Society for Medical Oncology. 2014;25(10):1930-4.

51. Egger M, Zellweger-Zahner T, Schneider M, Junker $C$, Lengeler $C$, Antes $G$. Language bias in randomised controlled trials published in English and German. Lancet (London, England). 1997;350(9074):326-9.

\section{Publisher's Note}

Springer Nature remains neutral with regard to jurisdictional claims in published maps and institutional affiliations. 